\title{
Crisis teams: systematic review of their effectiveness in practice
}

\author{
Rebecca A. Carpenter, ${ }^{1,2}$ Jara Falkenburg, ${ }^{1,2}$ Thomas P. White, ${ }^{2}$ Derek K. Tracy ${ }^{1,2}$
}

The Psychiatrist (2013), 37, 232-237, doi: 10.1192/pb.bp.112.039933

${ }^{1}$ Oxleas NHS Foundation Trust, London ${ }^{2}$ Institute of Psychiatry, King's College London

Correspondence to Derek K. Tracy (derek.tracy@oxleas.nhs.uk)

First received 2 May 2012, final revision 28 Feb 2013, accepted 8 Mar 2013
Aims and method Crisis resolution and home treatment teams (variously abbreviated to CRTs, CRHTTs, HTTs) were introduced to reduce the number and duration of in-patient admissions and better manage individuals in crisis. Despite their ubiquity, their evidence base is challengeable. This systematic review explored whether CRTs: (a) affected voluntary and compulsory admissions; (b) treat particular patient groups; (c) are cost-effective; and (d) provide care patients value.

Results Crisis resolution teams appear effective in reducing admissions, although data are mixed and other factors have also influenced this. Compulsory admissions may have increased, but evidence that CRTs are causally related is inconclusive. There are few clinical differences between 'gate-kept' patients admitted and those not. Crisis resolution teams are cheaper than in-patient care and, overall, patients are satisfied with CRT care.

Clinical implications High-quality evidence for CRTs is scarce, although they appear to contribute to reducing admissions. Patient-relevant psychosocial and longitudinal outcomes are under-explored.

Declaration of interest D.K.T. works and R.A.C. and J.F. have worked in a crisis team. The team was one of the Royal College of Psychiatrists' successful pilot sites for the new Home Treatment Accreditation Scheme (HTAS).
There has been a move to community-based mental healthcare for the past half-century: ${ }^{1}$ the number of in-patient psychiatric beds in the UK fell from 152000 in 1954 to 29802 in $2005 .^{2}$ Crisis resolution and home treatment teams (known variously as CRHTTs, CRTs and HTTs; CRT will be used throughout this article) were established in the 1980s, but the first policy, mandated in The NHS Plan, did not come about until 2000. ${ }^{3}$ By 2006, 343 teams had been introduced. ${ }^{2}$ They were designed as ward 'gatekeepers', providing intensive short-term care to vulnerable patients considered for admission or discharge. ${ }^{4}$ Teams of approximately 14 multidisciplinary staff, predominantly nursing, were anticipated to have caseloads of 20-30 and to be available 24 hours a day, 7 days a week, to a wide variety of patients. Potential interventions would include assessment and engagement of patients in crisis, psychological support and education, medication review and administration, and social support and advocacy.

Despite these laudable aims, it has been argued that policy was implemented without sufficient evidence. ${ }^{5,6}$ To date there has been little direct advice or guidance specifically to CRTs on markers of good care or outcomes. The Royal College of Psychiatrists is attempting to redress this with the recent piloting of the Home Treatment Accreditation Scheme (HTAS) that will, following refinement of goals through analysis of data obtained in this pilot, establish national standards for accredited teams. In this context of a questionable research base and the setting up of a national accreditation scheme, and under the spectre of future primary care (de)commissioning of services, we have set out to systematically review the existing evidence for CRTs and to provide a commentary on this. In particular, this review aims to establish: (a) whether CRTs have affected voluntary and/or compulsory admissions; (b) the clinical profiles of patients admitted despite CRTs; (c) whether CRTs are cost-effective; and (d) whether patients are satisfied with the care received.

\section{Method}

\section{Search strategy}

A systematic literature review was conducted and reviewed by R.A.C. and D.K.T. References were retrieved via PubMed and PsycINFO (1998-present) as well as through searches of studies in recent reviews ${ }^{1,7,8}$ and scanning reference lists of articles found: the keywords used were 'psychiatr" ${ }^{*}$ or 'mental', and 'crisis resolution' or 'crisis assessment' or 'crisis intervention' or 'intensive home treatment' or 'home treatment'. The last search was run on 29 March 2012.

\section{Eligibility criteria}

The following exclusion criteria were established in advance of the literature search. 
- Participants: studies that looked specifically at children and adolescents, older adults or only staff were excluded, thus focusing only on working-age adults, for whom CRTs were intended. ${ }^{3}$

- Interventions: only studies that described a CRT as defined in The NHS Plan, ${ }^{3}$ and therefore only those published after 2000.

- Comparators: no comparators were required for inclusion in this systematic review.

- Outcomes: studies that addressed one or more of the research questions, that is: (a) voluntary and/or compulsory admission rates and duration of admission either between an area with a CRT compared with an area without, or pre- and post-CRT introduction in a certain catchment area; (2) the characteristics of patients admitted to hospital since CRT introduction; (c) the cost-effectiveness of CRTs; and (d) quantitative and qualitative measures of patient satisfaction with CRT care.

- Study design: both randomised controlled trials (RCTs) and non-RCTs were reviewed.

Studies that were not published in English were excluded.

\section{Study selection}

The initial search of electronic databases produced 2169 abstracts. First, these were sequentially screened on the basis of title and abstract and 1983 articles that clearly did not refer to a CRT were excluded. Second, 93 duplicates were removed by importing all remaining articles into an Excel file and matching titles, authors' names and sources. At this stage, there were 79 articles that were read in full and those that did not meet the eligibility criteria were excluded. The reference lists of all articles that were reviews or meta-analyses were examined and this revealed 4 articles that had not been found in the initial literature search, such that 83 articles were read in full. Of these 83,10 studies were excluded because they did not meet the participants' eligibility criteria, 16 because they did not meet the intervention's eligibility criteria, 18 because they did not meet the outcome's eligibility criteria, and 2 studies were excluded because of duplicate publication (same content but different title and journal). Ultimately, 37 articles were reviewed. A flow diagram summarising the study selection process is shown in Fig. 1.

\section{Data extraction}

We extracted the data to collate all the relevant information from each article: year and location of publication, study type, number and characteristics of participants, primary and secondary outcome measures, primary and secondary results and limitations of study.

\section{Data analysis}

For each of the four review aims, a table is presented with characteristics of included studies, primary outcome and risk of bias within the studies. Meta-analyses generally present an elegant means of summarising the existing literature. However, the means in which previously published findings are reported prevented meta-analysis from being usefully conducted for the purposes of this work. For both cost-effectiveness and patient satisfaction only one study in each provided sufficient detail for inclusion into a meta-analysis, and thence this was not carried out.

\section{Results}

\section{Have CRTs affected voluntary and compulsory in-patient admissions?}

\section{Voluntary admission rates and duration}

The NHS Plan ${ }^{3}$ stated that CRTs should produce a $30 \%$ reduction of both rate and length of admission. Twenty studies, of varying quality, addressed this question (see online Table DS1 for characteristics of included studies and risk of bias within studies), but, perhaps due to the inherent ethical and clinical problems of randomisation in such a vulnerable population, only one was an RCT. ${ }^{9}$ This RCT compared a CRT in inner London with standard care, consisting of in-patient services, local community mental health teams and two crisis houses. Patients receiving care from a CRT were less likely than controls to be admitted at the 8-week and 6-month study points, but compulsory admissions were not significantly reduced. By 6 months, the number of days spent in hospital was also statistically less in the CRT group, but the severity of clinical and social outcomes was similar between groups. The study was limited in that 104 admitted patients were excluded from the analysis for several different reasons, including patients not following study procedures $(n=25)$ and incapacitous or non-consenting patients $(n=46)$ : inevitably, such patients were likely to be more disturbed than average but represent part of a typical CRT case-load.

Twelve 'pre- and post-CRT comparison' studies compared outcomes before and after the introduction of a CRT, with eight studies showing reduced admission rates post-introduction and four reporting no difference. One study found statistically significant reductions in admission rates $(71 \% v .49 \%)$ and mean number of bed days (19.1 v. 12.9) in the 6 weeks following a crisis. ${ }^{10}$ Likewise, another study $^{11}$ showed a $24 \%$ decrease in acute psychiatric admissions, a $22 \%$ decrease in mean duration of in-patient stay, a $17 \%$ reduction in Mental Health Act admissions, and a $4 \%$ decrease in readmissions. Keown and colleagues ${ }^{12}$ found admission rates decreased by $45 \%$ after CRT implementation, and more so for females and younger adults: they postulated this was because younger women may have more support in the community or stay out of hospital for childcare. This research also found that the length of stay increased from 15.5 to 25 days after CRT implementation.

Conversely, two studies, one in Australia ${ }^{13}$ and one in Scotland, ${ }^{14}$ found no statistically significant differences in admission rates, although both CRTs studied experienced staff shortages. A study of a CRT in Wales ${ }^{15}$ found overall bed usage unchanged, attributed to a reduction in informal admissions coinciding with compulsory admissions increasing. Similarly, another study, in England, found no differences, although it lacked statistical power. ${ }^{16}$

Interpreting admission rate and duration data These 'preand post-CRT' studies aid discussion but face the inherent confounders of temporally distinct assessments and the fact 


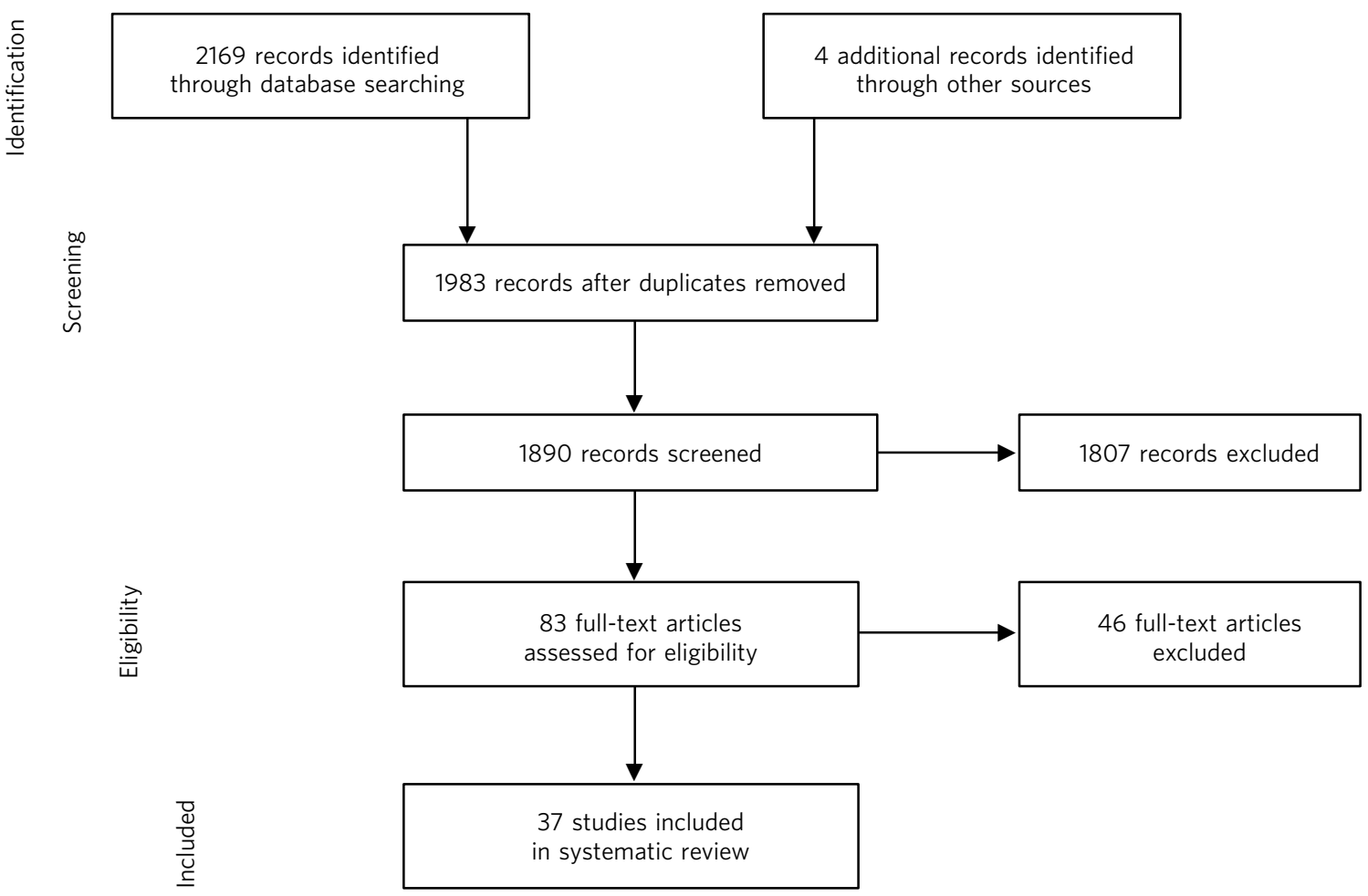

Fig 1 Flow diagram of study selection process.

that CRT introduction often coincides with bed reductions. ${ }^{15}$ In an examination of admission rates in 229 primary care trusts in England, of which 34 had CRTs, researchers ${ }^{17}$ found that admissions nationally fell $11 \%$ between 1998 and 2004. However, in the 34 primary care trusts that had CRTs, admissions had fallen by $10 \%$ more - and by $23 \%$ more in the 12 primary care trusts with teams that were on call 24 hours a day - although CRTs had not made a significant difference in bed use. Reductions in admission were most noticeable in more established teams and variability may also arise as, despite being conceptualised as gatekeeping all potential admissions, the National Audit Office found CRTs typically involved in only 53\% of admissions and had influenced the decision to admit in only $46 \% .^{18}$ Contemplating CRTs further reducing admissions must be contextualised by consideration that current bed numbers may be close to a practical minimum. ${ }^{19}$ An interesting finding was work showing a relationship between CRT introduction and increased length of admission. ${ }^{12}$ The interpretation of this is open to debate, but a posited explanation is that CRT gatekeeping means those actually admitted were typically more ill; alternatively, it is conceivable that fewer admissions resulted in less ward pressure to discharge.

Gatekeeping crisis admissions and risk Tyrer and colleagues $^{15}$ found a higher number of deaths by suicide in an area covered by a CRT compared with another area locally that had none, and compared with the same area 9 months before CRT introduction. Conversely, another study $^{12}$ found that suicide rates remained constant, concluding that CRTs are as safe as standard hospital care.
Whether CRTs, by keeping individuals in crisis in the community, have increased risk has been - surprisingly, given their role - under-explored.

\section{Involuntary admissions}

Since 1999, the number of detentions under the Mental Health Act 1983 has remained relatively stable ${ }^{20}$ - with some increase in Section 2 and reduction in Section 3 admissions - contrary to expectations that CRTs would decrease use of the Act. Nine studies investigating this have had mixed results, with two reporting a decrease, one of which was not significant; two no difference; and five reporting an increase (Table DS1). One study ${ }^{21}$ used focus groups to qualitatively explore this: staff inferred that if the CRT referred patients for a Mental Health Act assessment then the 'least restrictive option' had already been attempted, with the next logical option detention under the Act. Further, approved mental health professionals expressed increasing difficulty negotiating informal admissions with ward staff and felt compulsory admission was the only way to get the patient admitted. The decreased use of Section 3 may be related to the fact that CRTs facilitate early discharge, ${ }^{21}$ although changes in culture and practice of the Mental Health Act assessments may have contributed. Keown and colleagues ${ }^{22}$ reported that involuntary admissions had increased by $64 \%$, directly associated with a reduction by $62 \%$ in provision of beds: the authors suggested that non-availability of beds was causing delays in or unfeasibility of planned admissions, resulting in patients deteriorating to such an extent that they required compulsory admission. However, although this has also been suggested elsewhere, ${ }^{12,15}$ there is no current empirical evidence for this hypothesis. 


\section{Clinical profiles of patients admitted to hospital despite the introduction of CRTs}

Seven observational studies addressed this question (see online Table DS2 for characteristics of included studies). The most common reason for admission was risk to self. ${ }^{31-33}$ Brimblecombe and colleagues ${ }^{31}$ found that $21.1 \%$ of patients taken on by CRTs were later admitted: $53.2 \%$ due to risk to self; $11.3 \%$ due to risk to others; and $8.1 \%$ as carers could not cope. However, another study ${ }^{34}$ found those at risk of violence or unintentional self-harm through self-neglect or recklessness were more likely to be admitted than those at risk of 'deliberate' self-harm. Uncooperativeness was also cited as a reason for admission. ${ }^{32,34}$ Psychosis was associated with hospitalisation in some work, ${ }^{28,34}$ although others found no diagnostic differences, ${ }^{33,35}$ with one study ${ }^{33}$ also finding no significant differences in demographic variables in those admitted. Overall, research findings have been inconsistent.

\section{Are CRTs cost-effective?}

Eight studies addressed this question (see online Table DS3 for characteristics of included studies). One of these $\mathrm{s}^{36}$ re-analysed previously reported RCT data, ${ }^{9}$ three were 'pre- and post-CRT comparisons, ${ }^{26,27,37}$ one compared police and crisis handling of psychiatric emergencies, ${ }^{30}$ and one was a national audit using a decision model. ${ }^{38}$ Overall, as might be expected, the CRTs were less expensive than in-patient care, although no statistical testing was reported in some work. ${ }^{26,30}$ One 'pre- and post-CRT comparison' $^{30}$ found that the average case cost was US $\$ 1520$ for the crisis service and US\$1963 for the police intervention. The second study ${ }^{26}$ compared two 6-month periods before and after the introduction of a CRT, and found that in-patient care costs fell sufficiently to more than cover the small rise in emergency care costs. The third study ${ }^{27}$ found no difference overall with the lower cost per individual mitigated by more people receiving acute care.

A decision model was developed by the National Health Service to estimate the costs and potential savings of CRTs. ${ }^{38}$ This concluded that expected 28-day care costs were $£ 630$ less than if CRT treatment had not been considered. Given that roughly $50 \%$ of patients in crisis are considered for $\mathrm{CRT}^{18}{ }^{18}$ of whom approximately $69 \%$ are not admitted, ${ }^{38}$ estimated savings in the UK are $£ 66$ million per year. However, this model makes a number of simplifications and only applied to the period of the crisis, so the longer-term costs or savings are unknown.

Other work $^{36,37}$ investigated cost-effectiveness using previously collected data. ${ }^{9,10}$ Over a 6-month follow-up period CRT patients cost $£ 2438$ less than in-patients. ${ }^{36}$ In the prospective controlled trial ${ }^{37}$ the finding that a CRT patient costs less per 6 months than an in-patient was only significant when patients who had any CRT contact were compared with those that had no contact, rather than comparing pre- or post-CRT groups.

\section{Are patients satisfied with CRT care?}

Thirteen studies addressed this question (see online Table DS4 for characteristics of included studies): two systematic reviews, ${ }^{7,8}$ one $\mathrm{RCT}^{9}{ }^{9}$ six quantitative analyses, ${ }^{9,10,30,39-41}$ three qualitative analyses, ${ }^{42-44}$ and two using both. ${ }^{11,45}$ Some studies ${ }^{15,27}$ attempted to collect patient satisfaction data as secondary analyses, but had response rates considered too low to report given the issue of response bias.

All seven quantitative studies report overall positive patient experience, although the $\mathrm{RCT}^{9}$ showed only a trend and not a statistically significant difference between inpatient and CRT satisfaction. Johnson and colleagues ${ }^{10}$ reported a highly significant difference in patient satisfaction pre- and post-CRT implementation, and $\mathrm{Scott}^{30}$ found over $85 \%$ of patients were satisfied with CRT care. Ninetytwo per cent of patients who responded to a survey in one study said they would be prepared to use the service again. ${ }^{40}$ In another study, ${ }^{11} 93 \%$ of patients reported some clinical improvement during their CRT care, with $27 \%$ feeling totally recovered at discharge. The study also reported that $89 \%$ felt safe during CRT care and $78 \%$ felt ready to be discharged from care. In a study of ethnic variation in satisfaction, ${ }^{41}$ it was found that whereas both White and Asian patients preferred home treatment to admission, explanations for this varied. For example, Asian patients showed greater concerns about religious and dietary requirements as well as greater levels of stigma surrounding hospitalisation.

Qualitative studies inevitably provide more depth of experience. One systematic review ${ }^{8}$ clustered its findings into three factors: access and availability; being understood as a 'normal' person; and dealing with a crisis in an everyday context. Patients appreciated having easy and rapid access to a service 24 hours a day, 7 days a week. ${ }^{43,44}$ Lack of frequent or prompt communication, possibly due to demands on resources, can cause the patient much anxiety and distress. ${ }^{45}$ Two aspects of the service frequently referred to as important in interviews were the practical help the team provided and being available to talk. Practical help came in many different forms, including providing medication, helping with daily routines such as showering, doing food shopping for the patient, and help with housing difficulties such as domestic chores and coordinating and paying utility bills. Patients also reported valuing the availability of staff to talk, ${ }^{43-45}$ including providing advice, information about other services, emergency numbers, appointment details and psychoeducation. However, some patients reported not receiving enough information, ${ }^{44}$ or receiving too much information in the form of confusing, jargon-filled leaflets.

Patients appreciated it when staff were reassuring, 'like a friend', and not 'fazed' by anything, ${ }^{43}$ and establishing strong relationships with staff was deemed very important to most patients interviewed. ${ }^{11,43,44}$ Patients were grateful for the hope and encouragement the team provided. ${ }^{44}$ Two studies reported that patients appreciated being treated as individuals, with services flexible to their needs. ${ }^{43,44} \mathrm{~A}$ strong association was found between the level of personal engagement in the service and patient satisfaction with the service, and its absence was mostly reported in teams that lacked resources. $^{45}$

A stated detrimental feature to forming such good relationships was a lack of consistency in who visited the patients' homes. ${ }^{43-45}$ However, many patients found such inconsistency acceptable if staff were adequately briefed 
and worked well together as a team, ${ }^{44}$ with most patients understanding the nature of a shift-based service. Another concern was when patients felt the team was at full capacity, as time and resources, such as visits, could be rushed or they were given telephone calls instead. ${ }^{45}$ Patients also emphasised the need for good communication between them, the team, the social network, different staff members and different mental health teams. They appreciated when family were kept informed and were told how best to support them. ${ }^{43,44}$ They also appreciated when they were included in decisions, ${ }^{44,45}$ although another study found patients in crisis were less concerned about this as they were less capable of making decisions. ${ }^{43}$

Evidence suggests that patients prefer home treatment to hospital admission due to the stigma of the latter, ${ }^{44}$ and they can carry on with some day-to-day activities and find ways to deal with the crisis in the context of their own life situation. ${ }^{45}$ However, some patients felt they were discharged to the CRT before they were ready, or would have preferred hospitalisation because they felt unsafe, that they were putting too much strain on their family, or because the home environment was a contributing factor to their mental health problems. ${ }^{45}$ Khalifeh and colleagues ${ }^{42}$ assessed the impact of CRTs on mothers and their children: interestingly, although most mothers preferred home treatment because they felt better looked after at home, most children preferred hospital admission as it relieved them of distress and responsibility. Common difficulties reported by the mothers were with meeting the children's physical needs, being dependent on the children and children witnessing distressing symptoms. Despite this, mothers were commonly reluctant to seek help with parenting because of fears their children could be taken away.

One study ${ }^{43}$ found most patients least satisfied with the end of treatment, either because it happened too quickly or because of poor communication with the service they were being referred to. ${ }^{45}$ These problems were not universal, however, and most patients accepted the ending of CRT care as long as they were given sufficient warning, explanation and details when changes were going to occur, and a specific plan for what they should do if a crisis were to occur again.

\section{Discussion}

Crisis resolution teams were implemented in the UK based on projected reductions on hospital admissions - both rates and durations - and compulsory detentions, improved patient satisfaction and cost savings, but without adequate evidence that they would be effective in meeting these aims. This systematic review illustrates an overall trend of falling admission rates that is massively confounded by national changes in in-patient bed numbers, although rates fell more so for areas that had a $\mathrm{CRT}^{17}$ and the evidence overall supports a modest positive effect in reducing admission rates and duration. Data surrounding compulsory Mental Health Act admissions are similarly likely affected by cultural changes in practice, and evidence for the effects of CRTs on this is limited: although there is some conflict in research findings, more studies reported an increase in Mental Health Act admissions, certainly against hypothesised predictions. The most consistent findings are that patients are generally satisfied with the care received from CRTs and that such teams are cost-effective alternatives to hospital admission.

Despite their ubiquity, CRTs remain under-researched and the existing data highlight inherent difficulties undertaking research in CRTs, particularly a gold-standard RCT. Comparisons of temporally or geographically distinct datasets are reasonable initial attempts to circumvent this, but weaken findings, particularly against an acknowledged background of change of practices and resources. The availability of in-patient beds and the lowest safe and sustainable number that can and should be provided is an emotive and widely debated issue. Positive patient feedback and constructive criticism are to be welcomed but this must be contextualised by low return rates, inevitable bias in such samples, and a wider finding that the process of asking about 'service satisfaction' generally produces positive results.

Other difficulties interpreting the research include the fact that CRTs vary widely in the populations they serve, both sociodemographically and operationally through inclusion and exclusion criteria, and their relations and referral routes with primary care, in-patient wards, community teams and so forth. There is no agreement on the exact size, staffing profile or working hours for such teams, and even where and when they operate it is clear CRTs are not gatekeeping all admissions. Admission rates and duration are the most commonly studied factors, most likely as they are key conceptual functions of such teams as well as the fact that they are ostensibly 'easier' and more objective markers to evaluate, although the problems with this are noted above. Our inability to meta-analyse the existing data underscores the need to adhere to statistical conventions of presenting appropriate measures such as error measures. There is a stark lack of data on risk and longer-term outcomes: do CRTs longitudinally modify factors such as self-harm, readmission rates or social markers of functioning?

We are all entering a brave new world of primary care commissioning and payment by results. Presumed continuance of existing practice and staffing levels, particularly in the face of underwhelming evidence, is not viable. Difficulties with RCTs in emergency or crisis services are inherent, but there are ways around this, including active controls in a 'standard' $v$. 'enhanced' trial. Undertaking this, particularly in a larger multisite study, would allow an attempt to tease out team confounders around the nature of service provided, and patient confounders such as the nature and duration of illness and sociodemographic factors. Incorporated into this - or circumventing it completely through alternative naturalistic methodology longer-term follow-up studies are required.

\section{About the authors}

Rebecca A. Carpenter is an honorary researcher, Oxleas NHS Foundation Trust, and a graduate of the MSc in Mental Health Studies at the Institute of Psychiatry, London. Jara Falkenburg is an honorary researcher, Oxleas NHS Foundation Trust, and a graduate of the MSc in Mental Health Studies at the Institute of Psychiatry, London. Thomas P. White is a post-doctoral researcher at the Cognition, Schizophrenia and Imaging Laboratory, Institute of Psychiatry, London. Derek K. Tracy is a consultant psychiatrist, Oxleas NHS Foundation Trust, and a researcher at the Cognition, Schizophrenia and Imaging Laboratory, Institute of Psychiatry, London. 


\section{References}

1 Sjølie $\mathrm{H}$, Karlsson $\mathrm{B}, \mathrm{Kim} \mathrm{H}$. Crisis resolution and home treatment structure, process, and outcome - a literature review. J Psychiatr Ment Health Nurs 2010; 17: 881-92.

2 Crompton N, Daniel D. Guidance Statement on Fidelity and Best Practice for Crisis Services. National Institute for Mental Health in England, Care Services Improvement Partnership, 2007.

3 Department of Health. The NHS Plan: A Plan for Investment, A Plan for Reform. TSO (The Stationery Office), 2000

4 Department of Health. The Mental Health Policy Implementation Guide. Department of Health, 2001.

5 Pelosi AJ, Jackson GA. Home treatment - engimas and fantasies. BMJ (Clin Res ed) 2000; 320: 308

6 Smyth M, Hoult J. The home treatment enigma. BMJ 2000; 320: 305.

7 Hubbeling D, Bertram R. Crisis resolution teams in the UK and elsewhere. J Ment Health 2012; 21: 285-95

8 Winness MG, Borg M, Kim HS. Service users' experiences with help and support from crisis resolution teams: a literature review. J Ment Health 2010; 19: 75-87.

9 Johnson S, Nolan F, Pilling S, Sandor A, Hoult J, McKenzie N, et al. Randomised controlled trial of acute mental health care by a crisis resolution team: the north Islington crisis study. BMJ 2005; 331: 599.

10 Johnson S, Nolan F, Hoult J, White IR, Bebbington P, Sandor A, et al. Outcomes of crises before and after introduction of a crisis resolution team. Br J Psychiatry 2005; 187: 68-75.

11 Barker V, Taylor M, Kader I, Stewart K, Le Fevre P. Impact of crisis resolution and home treatment services on user experience and admission to psychiatric hospital. Psychiatrist 2011; 35: 106-10.

12 Keown P, Tacchi MJ, Niemiec S, Hughes J. Changes to mental healthcare for working age adults: impact of a crisis team and an assertive outreach team. Psychiatr Bull 2007; 31: 288-92.

13 Adesanya A. Impact of a crisis assessment and treatment service on admissions into an acute psychiatric unit. Australas Psychiatry 2005; 13: 135-9.

14 Forbes NF, Cash HT, Lawrie SM. Intensive home treatment, admission rates and use of mental health legislation. Psychiatrist 2010; 34: 522-4.

15 Tyrer P, Gordon F, Nourmand S, Lawrence M, Curran C, Southgate D, et al. Controlled comparison of two crisis resolution and home treatment teams. Psychiatrist 2010; 34: 50-4

16 Jacobs R, Barrenho E. Impact of crisis resolution and home treatment teams on psychiatric admissions in England. Br J Psychiatry 2011; 199 $71-6$.

17 Glover G, Arts G, Babu KS. Crisis resolution/home treatment teams and psychiatric admission rates in England. Br J Psychiatry 2006; 189: 441-5.

18 Morgan S. Are Crisis Resolution and Home Treatment Services Seeing the Patients they are Supposed to See? National Audit Office, 2007

19 Lelliott P. Acute inpatient psychiatry in England: an old problem and a new priority. Epidemiol Psichiatr Soc 2006; 15: 91.

20 Department of Health. Department of Health Autumn Performance Report 2006. TSO (The Stationery Office), 2006

21 Furminger $E$, Webber $M$. The effect of crisis resolution and home treatment on assessments under the 1983 Mental Health Act: an increased workload for approved social workers? Br J Soc Work 2009; 39: 901-17.

22 Keown P, Weich S, Bhui KS, Scott J. Association between provision of mental illness beds and rate of involuntary admissions in the NHS in England 1988-2008: ecological study. BMJ 2011; 343: d3736.

*23 Robin M, Bronchard M, Kannas S. Ambulatory care provision versus first admission to psychiatric hospital: 5 years follow up. Soc Psychiatry Psychiatr Epidemiol 2008; 43: 498-506.

*24 Uddin MS, Byrt R. Resolving crisis within the community. J Comm Nurs 2007; 5: 4-13.

*25 Jethwa K, Galappathie N, Hewson P. Effects of a crisis resolution and home treatment team on in-patient admissions. Psychiatr Bull 2007; 31: $170-2$
26 Damsa C, Hummel C, Sar V, Di Clemente T, Maris S, Lazignac C, et al. Economic impact of crisis intervention in emergency psychiatry: a naturalistic study. Eur Psychiatry 2005; 20: 562-6.

27 Ford R, Minghella E, Chalmers C, Hoult J, Raftery J, Muijen M. Cost consequences of home-based and in-patient-based acute psychiatric treatment: results of an implementation study. J Ment Health 2001; 10: 467-76

28 Guo S, Biegel DE, Johnsen JA, Dyches H. Assessing the impact of community-based mobile crisis services on preventing hospitalization. Psychiatr Serv 2001; 52: 223-8.

*29 Dunn LM. Mental Health Act assessments: does a community treatment team make a difference? Int J Soc Psychiatry 2001; 47: 1-19.

30 Scott RL. Evaluation of a mobile crisis program: effectiveness, efficiency, and consumer satisfaction. Psychiatr Serv 2000; 51: 1153-6.

31 Brimblecombe N, O'Sullivan G, Parkinson B. Home treatment as an alternative to inpatient admission: characteristics of those treated and factors predicting hospitalization. J Psychiatr Ment Health Nurs 2003; 10 683-7.

32 Brooker C, Ricketts T, Bennett S, Lemme F. Admission decisions following contact with an emergency mental health assessment and intervention service. J Clin Nurs 2007: 16: 1313-22.

33 Tomar R, Brimblecombe N, O'Sullivan G. Service innovations. Home treatment for first-episode psychosis. Psychiatr Bull 2003; 27: 148-51.

34 Cotton MA, Johnson S, Bindman J, Sandor A, White I, Thornicroft G, et al. An investigation of factors associated with psychiatric hospital admission despite the presence of crisis resolution teams. BMC Psychiatry 2007; 7: 52.

35 Harrison J, Alam N, Marshall J. Home or away: which patients are suitable for a psychiatric home treatment service? Psychiatr Bull 2001; 25: $310-3$.

36 McCrone P, Johnson S, Nolan F, Pilling S, Sandor A, Hoult J, et al. Economic evaluation of a crisis resolution service: a randomised controlled trial. Epidemiol Psichiatr Soc 2009; 18: 54

37 McCrone P, Johnson S, Nolan F, Pilling S, Sandor A, Hoult J, et al. Impact of a crisis resolution team on service costs in the UK. Psychiatr Bull 2009; 33: 17-9.

38 McCrone P, Knapp M, Hudson J. Model to Assess the Economic Impact of integrating CRHT and Inpatient Services. Centre for the Economics of Mental Health, King's College London, 2007.

39 Ruggeri M, Salvi G, Perwanger V, Phelan M, Pellegrini N, Parabiaghi A. Satisfaction with community and hospital-based emergency services amongst severely mentally ill service users. Soc Psychiatry Psychiatr Epidemiol 2006; 41: 302-9.

40 Kalucy R, Thomas L, Lia B, Slattery T, Norris D. Managing increased demand for mental health services in a public hospital emergency department: a trial of 'Hospital-in-the-Home' for mental health consumers. Int J Ment Health Nurs 2004; 13: 275-81.

41 Khan I, Pillay K. Users' attitudes towards home and hospital treatment: a comparative study between South Asian and white residents of the British Isles. J Psychiatr Ment Health Nurs 2003; 10: 137-46.

42 Khalifeh $H$, Murgatroyd C, Freeman M, Johnson S, Killaspy H. Home treatment as an alternative to hospital admission for mothers in a mental health crisis: a qualitative study. Psychiatr Serv 2009; 60: 634-9.

43 Hopkins C, Niemiec S. Mental health crisis at home: service user perspectives on what helps and what hinders. J Psychiatr Ment Health Nurs 2007; 14: 310-8.

44 Goldsack S, Reet M, Lapsley H, Gingell M. Experiencing a RecoveryOriented Acute Mental Health Service: Home Based Treatment from the Perspectives of Service Users, Their Families and Mental Health Professionals. Mental Health Commission, New Zealand, 2005.

45 Clark F, Khattak S, Nahal J, Linde K. Crisis Resolution and Home Treatment: The Service User and Carer Experience. National Audit Office, 2008.

*Studies cited in online Table DS1. 